\title{
MALFORMAÇÃO DE CHIARI DO TIPO II SINTOMÁTICA
}

\author{
J. FRANCISCO SALOMÃO*, ANTÔNIO R. BELLAS**, RENÊ D. LEIBINGER**, \\ ANA PAULA A. BARBOSA**, MARIA ANNA P. B. BRANDÃO**
}

\begin{abstract}
RESUMO - Os autores analisam uma série de 17 crianças portadoras de mielodisplasias que desenvolveram sinais e sintomas da malformação de Chiari do tipo II. De acordo com a idade, dois grupos ficaram bem definidos: Grupo I, crianças no primeiro ano de vida, em que predominaram sinais e sintomas de comprometimento do tronco encefálico e nervos cranianos bulbares ( $\mathrm{n}=13$ ); Grupo II, composto por crianças com idade superior a um ano, em que as principais manifestações foram dor cervical e sinais cerebelares $(n=4)$. O resultado do tratamento cirúrgico nos dois grupos foi distinto: enquanto a mortalidade no Grupo I atingiu 46,1\%, nenhum paciente do Grupo II veio a falecer. O tratamento inicial consistiu na instalação ou revisão de derivação ventricular, sendo a descompressão crânio-vertebral reservada àqueles que não se beneficiaram com esses procedimentos. Os autores enfatizam a necessidade do imediato reconhecimento e tratamento do quadro, de modo a se obter resultados satisfatórios.
\end{abstract}

PALAVRAS-CHAVE: mielomeningocele, malformação de Chiari do tipo II, malformação de ArnoldChiari, desconpressão de fossa craniana posterior, hidrocefalia, paralisia de cordas vocais.

\section{Symptomatic Chiari type II malformation}

ABSTRACT - The Chiari type II malformation is the leading cause of death in infants with myelomeningocele. The authors report 17 cases of symptomatic Chiari type II malformation occurring in two distinct age dependent population. In Group I, 13 neonates and infants in the first year of life presented with cranial nerve and brain stem dysfunction characterized by vocal cord paralysis, apnea, dysphagia and laryngeal stridor. In Group II, 4 patients developed signs and symptoms after the first year of life. In this group, the presentation was more insidious and included neck pain and cerebellar manifestations. The surgical treatment consisted initially in shunt implantation or revision and when there was no improvement, posterior fossa decompression was performed. The response to the surgical treatment differed considerably between the two groups: older patients improved promptly after surgery and there was no casualties; in newborn and infants, especially those under 6 months of age, the mortality rate was $46.1 \%$. The authors stress that prompt diagnosis and surgical intervention should be performed in order to produce a favorable outcome.

KEY WORDS: myelomeningocele, Chiari type II malformation, Arnold-Chiari malformation, posterior fossa decompression, hydrocephalus, vocal cord paralysis.

A malformação de Chiari do tipo II (MChII) compõe o espectro das herniações cerebelares inicialmente descritas em 1883 por Cleland (de acordo com Koehler ${ }^{1}$ ) e, posteriormente, em 1891 e 1896, por Chiari (citado por Koehler ${ }^{1}$ e $\mathrm{Carmel}^{2}$ ). O epônimo Arnold-Chiari foi introduzido em 1907 por Schwalbe e Gredig (de acordo com Koehler ${ }^{1}$ Carmel $^{2}$ ), discípulos de Arnold no laboratório de patologia de Heidelberg, para caracterizar as herniações cerebelares associadas à spina bifida cística. Alguns autores ${ }^{1,2}$ consideram a participação de Arnold discreta, por ser limitada a um único caso, preferindo a denominação de malformação de Chiari do tipo II.

Serviço de Neurocirurgia Pediátrica, Departamento de Cirurgia Pediátrica, Instituto Fernandes Figueira Fundação Oswaldo Cruz (M.S. - Fiocruz), Rio de Janeiro RJ: *Chefe de Serviço; **Neurocirurgião. Aceite: 27outubro-1997.

Dr. J. Francisco Salomão - Av. N.S. Copacabana 749/604 - 22050-000 Rio de Janeiro RJ - Brasil. 
Conforme a descrição de Chiari, o tipo I consiste no deslocamento caudal das amígdalas cerebelares através do forâmen magno; o tipo II, na herniação das amígdalas, verme cerebelar, IV ${ }^{\circ}$ ventrículo e porção inferior do bulbo, através do forâmen occipital; o tipo III na herniação do cerebelo e tronco encefálico dentro de uma meningocele cervical alta sendo que, no tipo IV, há hipoplasia cerebelar sem herniação. Várias anomalias do sistema nervoso se associam à MChII e incluem, dentre outras, craniolacunia, alargamento do forâmen magno, hipoplasia de núcleos de nervos cranianos e olivas cerebelares, embicamento ("beaking") da placa tectal, poligiria, heterotopias, laminação desordenada, aumento da massa intermédia do tálamo, colpocefalia, ausência de septo pelúcido, migração superior do cerebelo pela incisura tentorial, hipoplasia da foice do cérebro e da tenda do cerebelo ${ }^{3-6}$.

Embora presente em todos os portadores de mielomeningocele, a malformação nem sempre é sintomática $^{5,7-9}$. Quando isso ocorre, porém, torna-se a maior responsável pela mortalidade em portadores de mielodisplasias ${ }^{4,10-14}$.

Neste estudo analisamos aspectos clínicos e cirúrgicos da malformação de Chiari do tipo II em uma população de portadores de mielodisplasias acompanhadas no Instituto Fernandes Figueira (M.S. - Fiocruz), Rio de Janeiro, R.J. (IFF-Fiocruz).

\section{CASUÍSTICA E MÉTODOS}

Foram revistos os prontuários médicos de 114 crianças portadoras de mielodisplasias, acompanhados pelo Serviço de Neurocirurgia Pediátrica do IFF-Fiocruz entre janeiro-1987 e dezembro-1996. Em todos os casos, avaliaram-se os dados referentes a sinais e sintomas relacionados à MChII, bem como a idade do paciente por ocasião do início do quadro, métodos diagnósticos empregados, procedimentos realizados, resposta ao tratamento e causa da morte. Os pacientes foram divididos em Grupos I (idade inferior a 12 meses) e Grupo II (idade superior a 12 meses).

Dentre 114 portadores de mielomeningocele, 17 (14,9\% ) exibiram sinais e sintomas atribuíveis à MChII, sendo 13 durante o primeiro ano de vida (Grupo I) e quatro após este período (Grupo II). Do total, 11 eram do sexo feminino e 6 do sexo masculino. O diagnóstico foi sempre estabelecido com base no quadro clínico. A tomografia computadorizada do crânio (TCC) foi realizada em todos os casos e ressonância nuclear magnética (RNM) do encéfalo e medula cervical, obtida em 6. Em nenhum caso, estes exames serviram para contra-indicar qualquer procedimento cirúrgico.

Em todos os casos do Grupo I , a sintomatologia desenvolveu-se entre 8 e 120 dias de vida (média 60,5 dias). Todos os pacientes tinham paralisia de cordas vocais, confirmada por laringoscopia, 7 pacientes apresentaram apnéia acompanhada de cianose, em 4, a primeira manifestação foi estridor laríngeo, 1 paciente desenvolveu tetraplegia (Tabela 1). Cinco eram previamente derivados e em 1, a TCC mostrou volumosa dilatação hidrosiringomiélica com extensão à fossa posterior, confirmada por ocasião da intervenção cirúrgica (Fig 1).

Tabela 1. Malformação de Chiari do tipo II no primeiro ano de vida: sinais e sintomas $(N=13)$.

\begin{tabular}{cc}
\hline Sinais e Sintomas & $\mathrm{N}^{\mathbf{o}}$ \\
\hline Paralisia de cordas vocais & 13 \\
Disfagia neurogênica & 11 \\
Crises de apnéia & 7 \\
Pescoço retrovertido & 5 \\
Opistótono & 2 \\
Apnéia sustentada & 1 \\
Tetraplegia & 1 \\
\hline
\end{tabular}

Tabela 2. Malformação de Chiari do tipo II após o primeiro ano de vida: sinais e sintomas $(N=4)$.

\begin{tabular}{cc}
\hline Sinais e Sintomas & $\mathrm{N}^{\mathrm{o}}$ \\
\hline Dor cervical & 3 \\
Nistagmo & 2 \\
Espasticidade & 1 \\
Disbasia & 1 \\
Sinais bulbares & 1
\end{tabular}




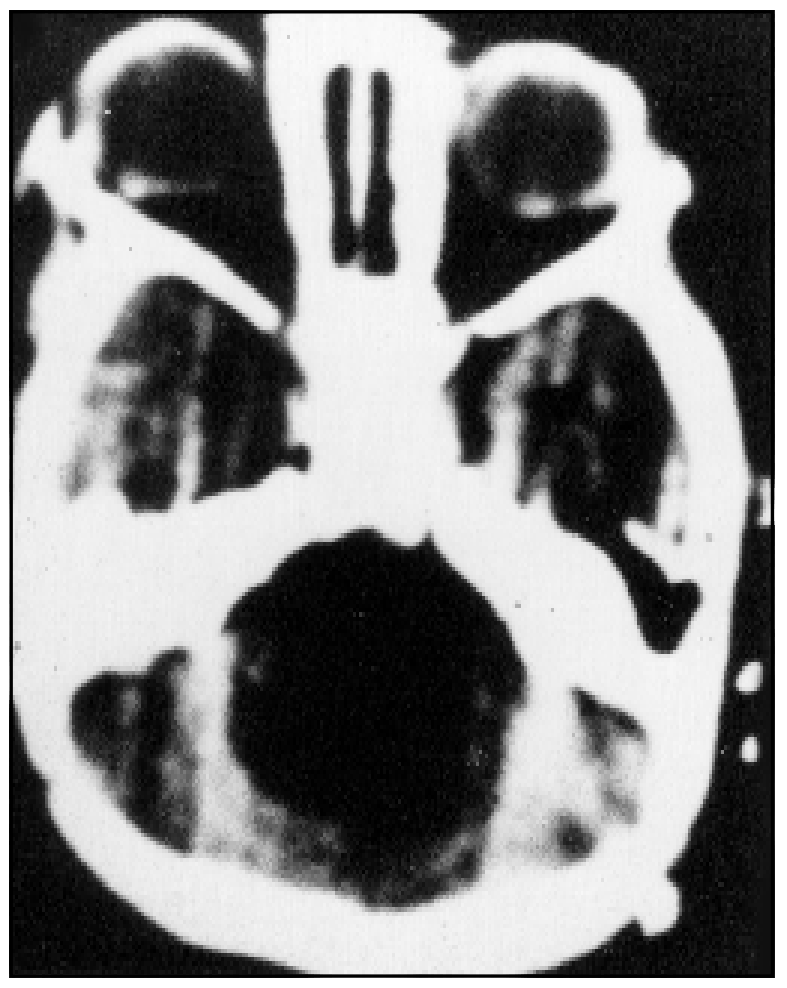

Fig 1. Malformação de Chiari do tipo II sintomática. TCC mostrando dilatação cística da fossa posterior, correspondendo a hidro-siringomielia com extensão ao bulbo raquídeo.

Fig 2. Lactente sintomático devido a posicionamento defeituoso do catéter proximal do sistema de derivação ventricular.

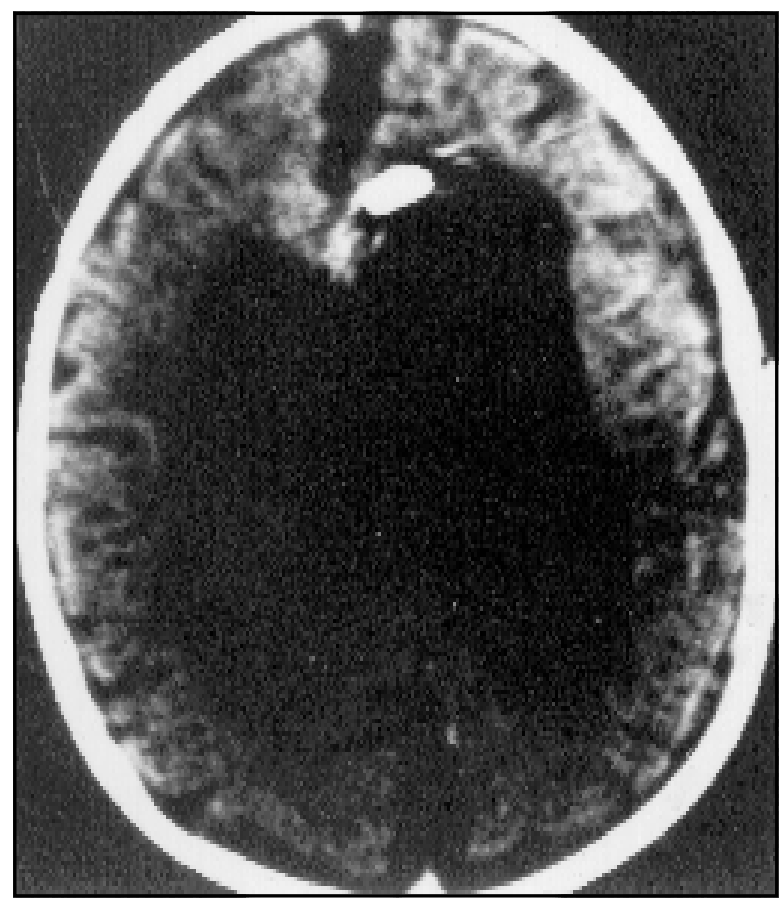




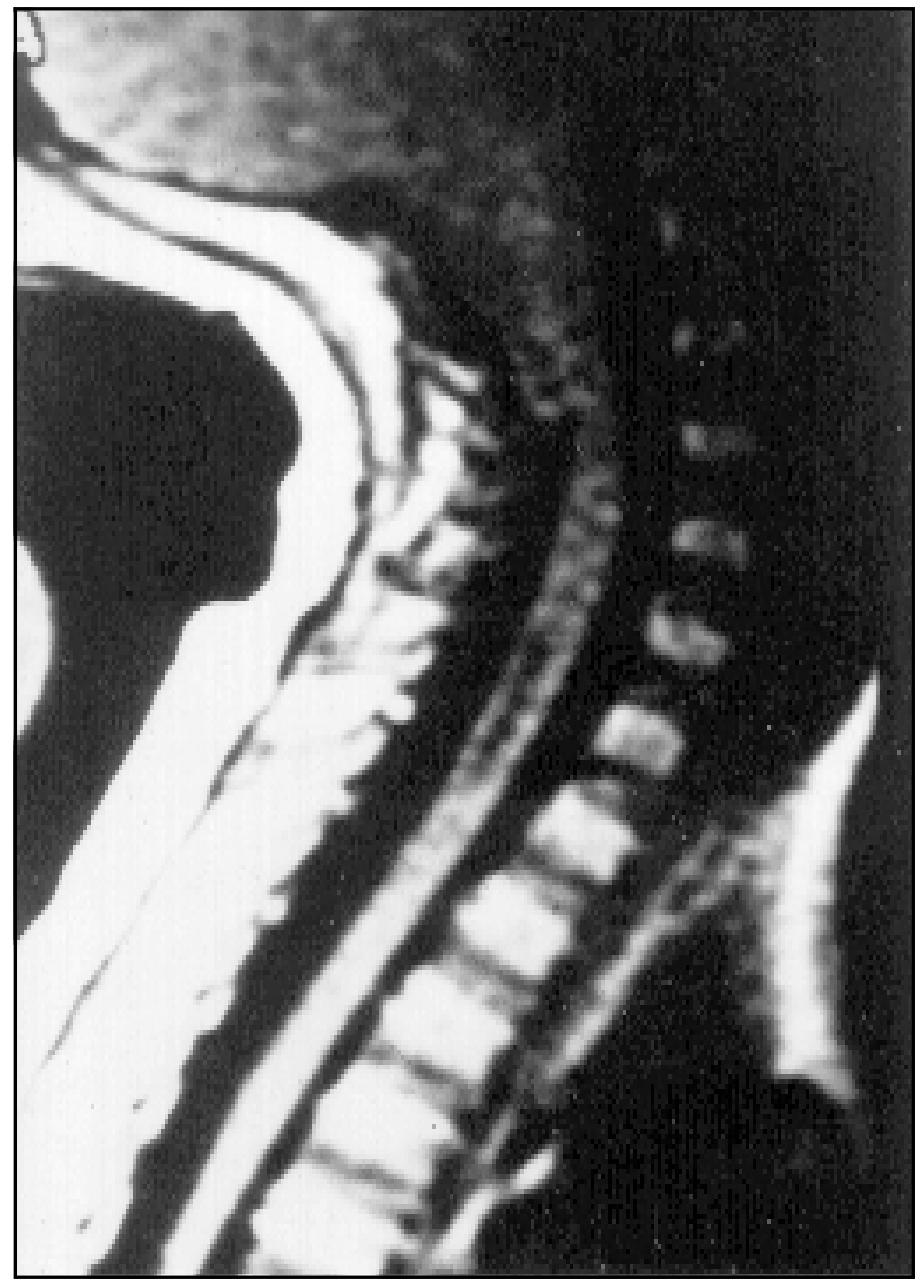

Fig 3. Mesmo caso da Figura 2, nota-se herniação das amígdalas cerebelares e cavitação siringomiélica na região cervical.

No Grupo II, a evolução foi mais insidiosa, com sinais e sintomas se manifestando entre 14 meses e 6 anos de idade (média 3,8 a). Em 3 pacientes as queixas foram dores na região cervical e limitação dos movimentos do pescoço, o quarto apresentava frequentes crises de opistótono, 2 pacientes apresentavam nistagmo, um tinha espasticidade com hiperreflexia nos membros superiores, enquanto outro tinha acentuada disbasia, 1 paciente tinha disfagia e regurgitação de alimentos pelas fossas nasais (Tabela 2). Dois pacientes deste grupo tinham disfunção do sistema de derivação.

Pacientes com hidrocefalia não tratada foram inicialmente submetidos a derivação ventrículo-peritoneal. Nos previamente derivados, a primeira medida foi certificar-se que o sistema funcionava adequadamente. As alternativas terapêuticas empregadas podem ser analisadas na Tabela 3. Em 8 pacientes o tratamento definitivo consistiu em implantação de sistema de derivação ventricular; em três em revisão do mesmo (Fig 2 e 3 ) e, nos outros 6, em descompressão crânio-vertebral. Estes últimos haviam sido previamente derivados e os ventrículos encontravam-se drenados, sem que nenhuma melhora fosse observada (Fig 4 e 5). A descompressão crâniovertebral consistiu na remoção de variável número de lâminas, de modo a expor todo o cerebelo herniado no canal cervical. O limite superior da descompressão foi o forâmen magno, que se encontrava invariavelmente alargado. Em todos os casos, uma banda fibrosa foi identificada comprimindo a dura-máter no nível de C1. Em 
um caso havia ausência de arcos posteriores em praticamente toda a extensão do defeito, sendo a compressão exercida quase exclusivamente pela banda fibrosa. A inspeção do conteúdo dural revelou aracnoidite de variada intensidade, em todos os casos. Em um deles, já mencionado, o procedimento foi complementado com derivação siringo-subaracnóidea. A descompressão foi completada com enxerto de fáscia.

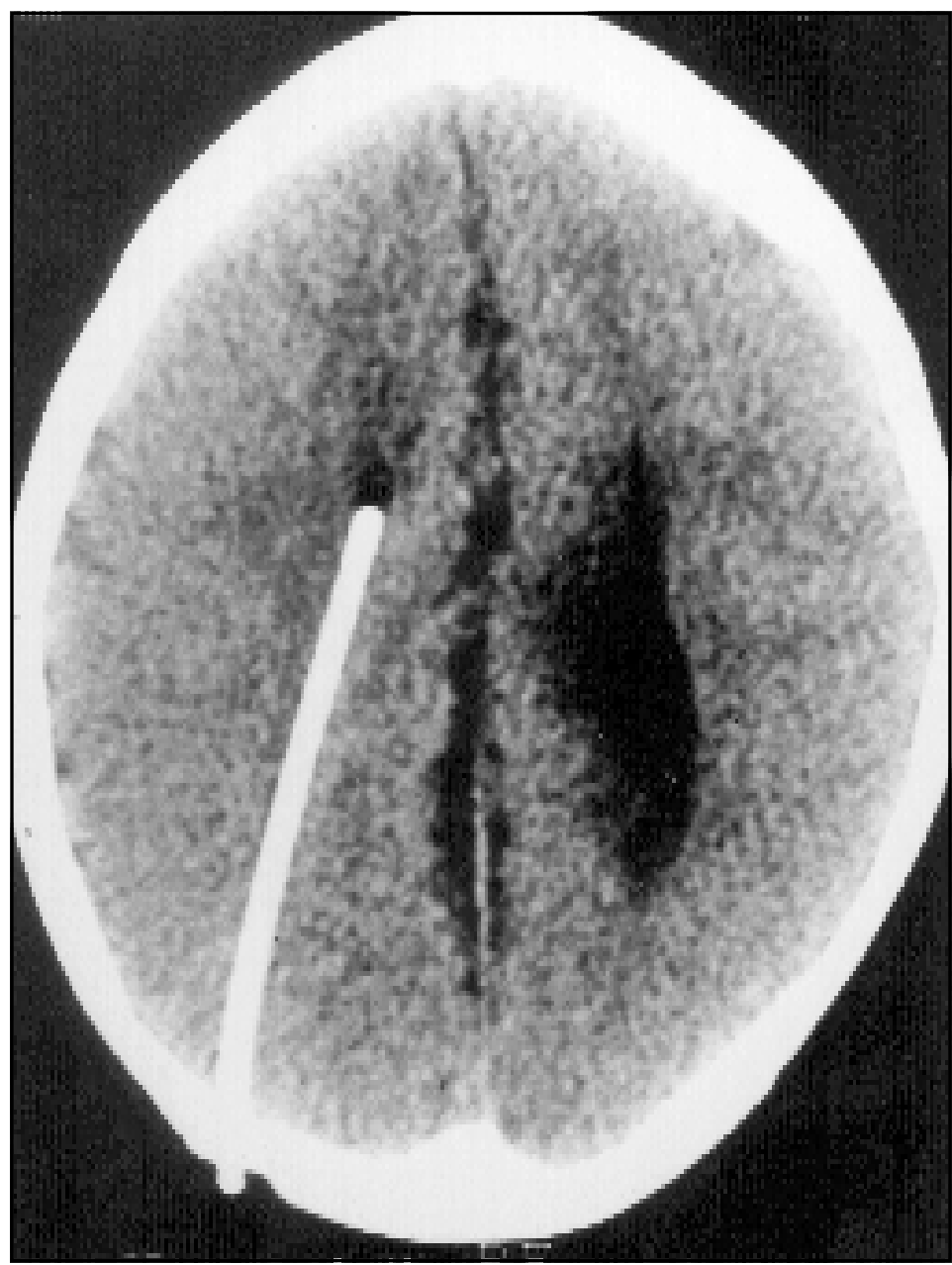

Fig 4. Lactente sintomático a despeito do funcionamento adequado do sistema de derivação ventrículo-peritoneal.

Tabela 3. Malformação de Chiari do tipo II sintomática: tratamento definitivo e resultados $(N=17)$

\begin{tabular}{ccccc}
\hline & \multicolumn{2}{c}{ Grupo I } & \multicolumn{2}{c}{ Grupo II } \\
& Pacientes & Óbitos & Pacientes & Óbitos \\
\hline Derivação ventricular & 8 & 3 & - & - \\
Descompressão de fossa posterior & 4 & 3 & 2 & - \\
Revisão de derivação & 1 & - & 2 & - \\
Total & 13 & 6 & 4 & - \\
\hline
\end{tabular}




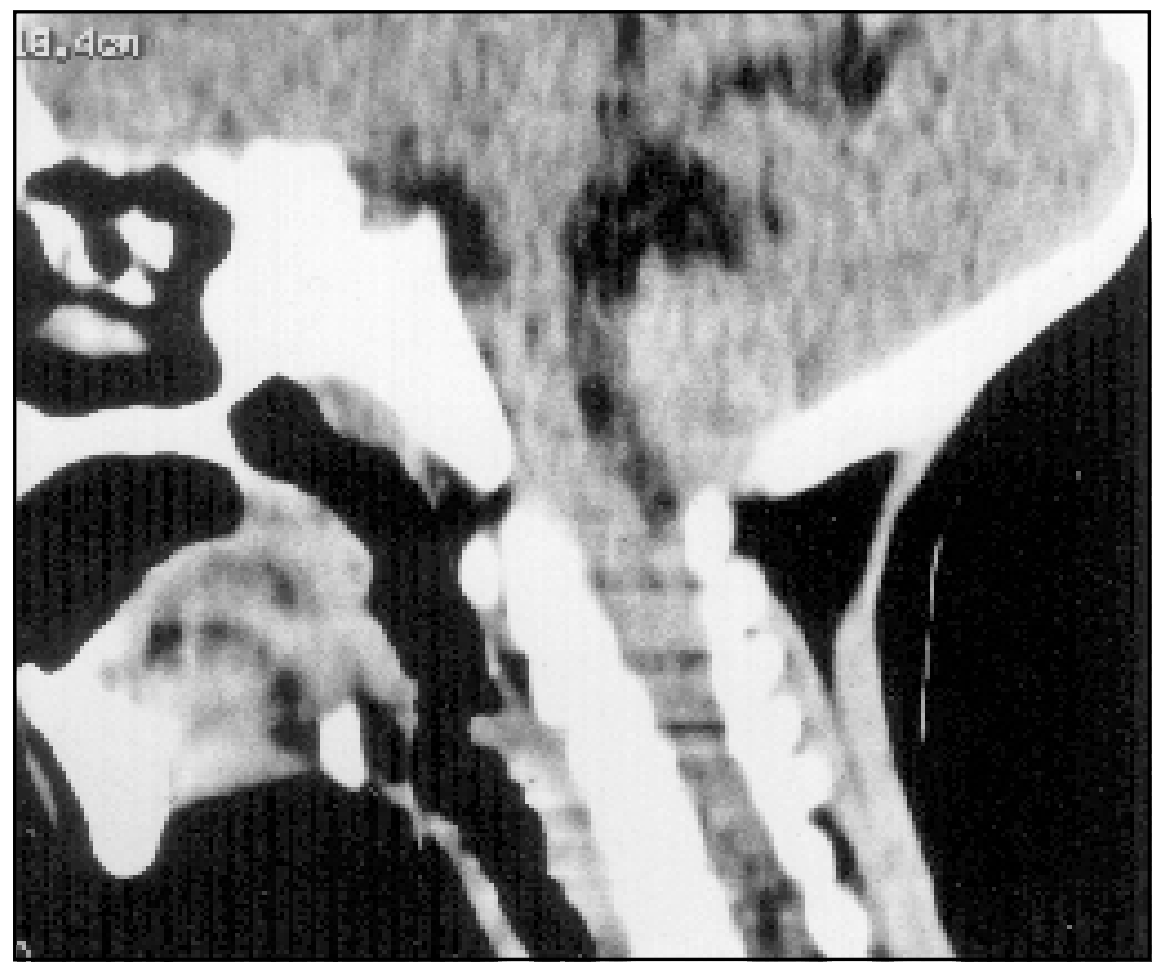

Fig 5. TCC em reconstrução tridimensional mostrando elementos da fossa craniana posterior herniados no canal cervical.

\section{RESULTADOS}

Todos os óbitos ocorreram no Grupo I. No total, 6 pacientes (35,3\% do total e $46,1 \%$ do Grupo I) faleceram. Dos 4 lactentes submetidos a descompressão da fossa posterior, 3 faleceram a despeito da melhora observada no pós-operatório imediato. Em todos os 3 houve ventriculite precedendo o quadro. Destes, 2 faleceram por complicações clínicas (septicemia por infecção urinária e pneumopatia) e o terceiro continuou apresentando crises esporádicas de apnéia, falecendo por ocasião de uma delas. Uma paciente encontra-se clinica e neurologicamente estabilizada, apesar de traqueostomizada. O intervalo de tempo entre o início do quadro e a descompressão crânio-cervical variou de 3 a 12 dias (media de 6,6 dias). Dos 8 pacientes submetidos a derivações ventriculares, 3 faleceram e, em apenas 1 , a causa da morte teve relação direta com a MChII (crise de apnéia). Nos outros 2, os óbitos relacionaram-se a complicações de derivações valvulares (hemorragia ventricular e ventriculite, respectivamente).

No Grupo II, todos os pacientes apresentaram imediata e dramática regressão dos sinais e sintomas, não se registrando óbitos ou complicações.

\section{DISCUSSÃO}

Penfield e Coburn 6 explicaram a patogenia da MChII como consequência da tração exercida pelo ancoramento medular no nível da mielomeningocele, o que, segundo Griebel et al. ${ }^{15}$, não justificaria as demais malformações associadas. A teoria hidrodinâmica de Gardner ${ }^{16-18}$ propõe que a imperfuração da fossa rombóide leve à distensão excessiva do tubo neural e consequente deslocamento caudal das estruturas da fossa posterior. Cameron ${ }^{3}$ sugere que a herniação ocorra em 
razão de um diferencial de pressão crânio-espinhal causada pelo escoamento de líquido

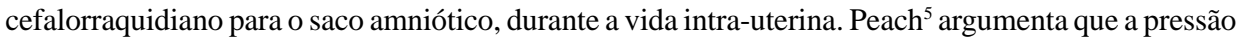
do líquido amniótico seria igualmente aplicada ao crânio e ao saco da mielomeningocele, motivo pelo qual este diferencial não existiria. McLone e Knepper ${ }^{19}$ acreditam que a malformação de Chiari tenha origem na neurulação defeituosa e que a incapacidade em manter a distensão do sistema ventricular primitivo seria responsável pela falta de indução mesenquimal, gerando uma fossa posterior de pequenas dimensões, incapaz de acomodar suas estruturas.

A MChII é a responsável pela maioria dos óbitos em portadores de mielomeningocele ${ }^{4,10,-14}$. Suas manifestações clínicas são insuficientemente conhecidas e nem sempre reconhecidas. Quando o são, sua importância é muitas vezes negligenciada ou subestimada, pois ocorrem em portadores de malformações complexas, de difícil tratamento e resultados pouco gratificantes. A incidência dos sinais e sintomas a ela atribuídos varia de acordo com as fontes consultadas. McLone ${ }^{12}$ admite que $32 \%$ dos portadores de mielomeningocele tenham, em algum momento, manifestações relacionadas à MChII. Para McCullough e Johnson ${ }^{20}$, o número dos que necessitam especial atenção para este problema não excederia $6 \%$. A MChII pode se manifestar em qualquer idade, e é mais severa em recém-nascidos e lactentes com menos de um ano de vida ${ }^{21,22}$, sendo melhor a resposta ao tratamento em crianças maiores e adolescentes ${ }^{9,22,23}$. Estas características são bastante evidentes em nossa casuística, em que todos os óbitos ocorreram em pacientes com idade inferior a 6 meses.

As manifestações clínicas costumam aparecer após a segunda semana de vida ${ }^{14,15,24,25}$, embora tenham sido também descritas já por ocasião do nascimento ${ }^{6}$. Em crianças com idade inferior a 1 ano predominam as manifestações relacionadas ao comprometimento do bulbo e dos nervos cranianos baixos. Paralisia de cordas vocais e estridor laríngeo costumam ser as mais frequentes manifestações clínicas. Dificuldade de sucção, apnéia, cianose, pescoço retrovertido e opistótono são frequentemente observados. Disfagia neurogênica resulta da compressão do tronco encefálico e nervos cranianos baixos, à qual invariavelmente se seguem outros sinais de comprometimento bulbar. Em recémnascidos, estes sintomas podem se manifestar por dificuldade de ingerir leite materno, mamada prolongada e ineficaz, acompanhada de tosse, regurgitação nasal, cianose e sinais de asfixia. Refluxo gastro-esofágico, regurgitação de alimentos e secreções, bem como perda dos reflexos orofaríngeo e traqueo-brônquico, predispõem a pneumonias recidivantes ${ }^{9,13,15,24,26}$. Os sinais e sintomas costumam ser mais severos em crianças com lesão lombossacra e motilidade preservada nos membros inferiores, que a início não apresentariam hidrocefalia importante ${ }^{13}$, escapando assim a eventuais critérios seletivos. Cochrane et al. ${ }^{27}$ dividem as crises apnêicas em dois grupos: as devidas a obstrução de vias aéreas e as consequientes a disfunção bulbar. No primeiro caso, a paralisia bilateral dos abdutores das cordas vocais seria responsável por respiração estertorosa e esforço respiratório, precedendo a parada respiratória. Esta forma de apnéia é aliviada por canulação endotraqueal ou traqueostomia. A apnéia mediada por mecanismos centrais também ocorre em associação a paralisia bilateral de cordas vocais, pode apresentar componente obstrutivo e se manifesta quando a criança é manipulada ou submetida a situações de desconforto emocional. Caracteristicamente, ocorre uma apnéia expiratória prolongada acompanhada por cianose que não costuma ser aliviada com as medidas acima descritas. Crianças mais velhas, adolescentes e adultos costumam apresentar sinais cerebelares, fraqueza nos membros superiores e espasticidade, sendo mais raro o acometimento de nervos cranianos ${ }^{11,15,24,28,29}$. O quadro neurológico é de evolver mais lento e menos ameaçador ${ }^{8}$.

Frequentemente, os sinais e sintomas são desencadeados por aumento da pressão intracraniana, seja por agravamento da hidrocefalia pré-existente, seja por disfunção de sistemas de derivação previamente implantados, como observado em alguns de nossos casos. Assim sendo, em nosso julgamento, o tratamento inicial deve sempre consistir em derivação valvular ou revisão do sistema já instalado, pois a sintomatologia pode desaparecer após controle da hidrocefalia ${ }^{2,8,15,27,30}$. A despeito destas medidas, o quadro pode evoluir e progressão da herniação tem sido demonstrada em RNM seriadas $^{31}$. Isto explica o aparecimento de sinais e sintomas em pacientes previamente estáveis, revelando o caráter progressivo da malformação. 
O tratamento da MChII que cursa com comprometimento de nervos cranianos é controverso, em grande parte pelos decepcionantes resultados observados em recém-nascidos e lactentes. A descompressão crânio-vertebral em pacientes de baixa idade é defendida por diversos autores s,21,23,32 $^{\text {. }}$ Vandertop et al. ${ }^{9}$ relataram mortalidade de $12 \%$ em 17 lactentes sintomáticos precocemente operados, sugerindo que uma pronta intervenção cirúrgica seria capaz de reverter o quadro. McLone ${ }^{12}$ coloca em questão o valor deste procedimento argumentando que muitos pacientes falecem e os que sobrevivem o fazem com acentuados problemas e qualidade de vida muito comprometida. Este autor acredita que para se defender a descompressão de fossa posterior em pacientes sintomáticos, um índice de sucesso superior a 2/3 deva ser demonstrado. Dahl et al. ${ }^{10}$ também registram prognóstico bastante desanimador, relatando a persistência de paralisia de cordas vocais, dificuldades de deglutição e necessidade de traqueostomia e gastrostomia, situação idêntica à por nós observada. Bell et al. ${ }^{24}$ registraram mortalidade de $50 \%$ em lactentes sintomáticos, concluindo que a descompressão cirúrgica tinha pouca influência na história natural da doença e que a inexorável progressão dos sintomas se relacionaria à desorganização intrínseca do tronco encefálico. McCullough e Johnson ${ }^{20}$ relatam que nenhum de seus pacientes foi submetido a descompressão: todos tinham derivações funcionantes, alguns necessitaram traqueostomia temporária e, os que evoluíram com estridor laríngeo, tiveram o problema contornado apenas com terapia respiratória. Milerad et al. ${ }^{33}$, relatam melhora gradual e posterior desaparecimento da apnéia do sono em lactentes tratados com acetazolamida, que estimularia os quimiorreceptores periféricos e centrais.

Alguns autores admitem que, nas formas sintomáticas da MChII, as causas sejam exclusivamente mecânicas ${ }^{9,21,34}$ e que a hidrocefalia controlada tornaria a compressão no nível da junção crânio-espinal o mais importante fator etiológico desta malformação. Em apoio a esta idéia, alterações isquêmicas e hemorrágicas atribuídas à compressão têm sido descritas ${ }^{21,34}$. Outros ${ }^{3-5,7,14,24}$ consideram que os sinais e sintomas estariam relacionados à desorganização estrutural do tronco encefálico, baseando-se em evidências clínicas e anatomopatológicas. Gozal et al. ${ }^{35}$, sugerem que os quimiorreceptores periféricos de portadores da MChII seriam anormais, tendo como consequência os distúrbios respiratórios associados ao sono, frequentemente observados nestes pacientes. Mori e Nishimura ${ }^{36}$ estudando potenciais evocados auditivos e somatosensitivos, observaram lenta maturação do tronco encefálico com sinais de deterioração, provavelmente resultando do estiramento de nervos cranianos baixos. Worley et al. ${ }^{37}$ identificaram potenciais evocados auditivos anormais em 11 dentre 12 neonatos que posteriormente desenvolveriam disfunção do tronco encefálico devido à MChII. Em 3 de nossos pacientes do Grupo I, os sinais e sintomas vieram a se manifestar após quadro de ventriculite e, apesar de os sistemas de derivação encontrarem-se funcionantes, houve progressão do quadro neurológico, sendo necessária descompressão crânio-cervical. A única paciente deste grupo que permanece viva foi aquela que não apresentou ventriculite. A nosso ver, a infecção do sistema nervoso pode ter adicionado um componente vascular à lesão compressiva do tronco encefálico, explicando assim sua progressão na vigência de pressão intracraniana controlada e a ausência de resposta à descompressão.

Torna-se óbvio que os resultados adversos obtidos na maioria de nossos lactentes submetidos a descompressão crânio-vertebral foram consequência da gravidade do quadro, refratário às outras medidas terapêuticas. Apesar das melhoras observadas, permaneceram com comprometimento de nervos cranianos bulbares, o que obrigou à realização de traqueostomia e gastrostomia e, em pelo menos um deles, a persistência de crises de apnéia foi a causa direta de sua morte.

\section{REFERÊNCIAS}

1. Koehler PJ. Historical vignette: Chiari's description of cerebellar ectopy (1891). With a summary of Cleland's and Arnold's contributions and some early observations on neural-tube defects. J Neurosurg 1991;75:823-826.

2. Carmel PW. Congenital syringomyelia. In Batzdorf U (ed). Syringomyelia: current concepts in diagnosis and treatment. Baltimore: Williams \& Wilkins, 1991:140-150.

3. Cameron AH. The Arnold-Chiari and other neuroanatomical malformations associated with spina bifida. J Pathol $1957 ; 73: 195-211$. 
4. Gilbert JN, Jones KL, Rorke LB, Chernoff GF, James HE. Central nervous system anomalies with associated meningocele hydrocephalus, and the Arnold-Chiari malformation: reappraisal of theories regarding the pathogenesis of posterior neural tube closure defects. Neurosurgery 1986;18:559-564.

5. Peach B. Arnold Chiari malformation: anatomic features of 20 cases. Arch Neurol 1965;12:613-621.

6. Penfield W, Coburn DF. Arnold-Chiari malformation and its operative treatment. Arch Neurol Psychiat 1938;40:328-336.

7. Charney EB, Rorke LB, Sutton LN, Schut L. Management of Chiari II complications in infants with myelomeningocele. J Pediatr 1987;111:364-371.

8. Rauzzino M, Oakes WJ. Chiari II malformation and syringomyelia. Neurosurg Clin N Am 1995;6:293-309.

9. Vandertop WP, Asai A, Hoffman HJ, et al. Surgical decompression for symptomatic Chiari II malformation in neonates with myelomeningocele. J Neurosurg 1992;77:541-544.

10. Dahl M, Ahlsten G, Carlson H, et al. Neurological dysfunction above cele level in children with spina bifida cystica: a prospective study to three years. Dev Med Child Neurol 1995;37:30-40.

11. Hahn YS. Open myelomeningocele. Neurosurg Clin N Am 1995;6:231-241.

12. McLone DG. Results of treatment of children born with a myelomeningocele. Clin Neurosurg 1983;30:407-435.

13. McLone DG. Continuing concepts in management of spina bifida. Pediatr Neurosurg 1992;18:254-256.

14. Sieben RL, Hamida MB, Shulman K. Multiple cranial nerve deficits associated with the Arnold-Chiari malformation. Neurology 1971;21:673-681.

15. Griebel ML, Oakes WJ, Morley G. The Chiari malformation associated with mielomeningocele. In Rekate HL (ed). Comprehensive management of spina bifida. Boca Raton: CRC Press, 1991:67-92.

16. Gardner WJ. Hydrodynamic mechanism of syringomyelia: its relationship to myelocele. J Neurol Neurosurg Psychiatry 1965;28:247-259.

17. Gardner WJ. Myelocele: rupture of the neural tube? Clin Neurosurg 1968;15:57-79.

18. Gardner WJ. The dysraphic states: from syringomyelia to anencephaly. Amsterdam: Excerpta Medica, 1973.

19. McLone DG, Knepper PA. The cause of the Chiari malformation: a unified theory. Pediatr Neurosci 1989;15:1-12.

20. McCullough DC, Johnson DL Myelomeningocele repair: technical considerations and complications. Concepts Pediat Neurosurg 1988;8:29-40.

21. Park TS, Hoffman HJ, Hendrick EB, Humphreys RP. Experience with surgical decompression of the Arnold-Chiari malformation in young infants with myelomeningocele. Neurosurgery 1983;13:147-152.

22. Venes JL. Multiple cranial nerve palsies in an infant with Arnold-Chiari malformation. Dev Med Child Neurol 1974;16:817-820.

23. Epstein F. Meningomyelocele: "pitfalls" in early and late management. Clin Neurosurg 1983;30:366-383.

24. Bell WO,. Charney EB, Bruce DA, Schut L. Symptomatic Arnold-Chiari malformation: review of experience with 22 cases. J Neurosurg 1987;66:812-816.

25. Wealthall SR, Whittaker GE, Greenwood N. The relationship of apnoea and stridor in spina bifida occulta to the unexplained infant's deaths. Dev Med Child Neurol 1974;16(Suppl):107-116.

26. Pollack IF, Pang D, Kocoshis S, Putnan P. Neurogenic dysphagia resulting from Chiari malformations. Neurosurgery 1992;30:709-719.

27. Cochrane DD, Addeley R, White CP, Norman P, Steinbok P. Apnea in patients with myelomeningocele. Pediatr Neurosurg 1991;16:232-239.

28. Carmel PW. Management of Chiari malformations in childhood. Clin Neurosurg 1983;30:385-406.

29. Hoffman HJ, Hendrick EB, Humphreys RP. Manifestations and management of Arnold-Chiari malformation in patients with myelomeningocele. Child's Brain 1975;1:255-259.

30. Haines SJ, Berger M. Current treatment of Chiari malformations types I and II: a survey of the pediatric section of the American Association of Neurological Surgeons. Neurosurgery 1991;28:353-357.

31. Ruge JR, Masciopinto J, Storrs BB, McLone DG. Anatomical progression of the Chiari II malformation. Child's Nerv Syst 1992;8:86-91.

32. Venes JL, Black KL, Latack JT. Preoperative evaluation and surgical management of the Arnold-Chiari II malformation. J Neurosurg 1986;64:363-370.

33. Milerad J, Lagercrantz H, Johnson P. Obstructive sleep apnea in Arnold-Chiari malformation treated with acetazolamide. Acta Paediatr 1992;81:609-612.

34. Morley AR. Laryngeal stridor, Arnold Chiari malformation and medullary haemorrhages. Dev Med Child Neurol 1969;11:471-474.

35. Gozal D, Arens R, Omlin KJ, Jacobs RA, Keens TG. Peripheral chemoreceptor function in children with myelomeningocele and Arnold-Chiari malformation type 2. Chest 1995;108:425-431.

36. Mori K Nishimura T. Electrophysiological studies on brainstem function in patients with myelomeningocele. Pediatr Neurosurg 1995;22:120-131.

37. Worley G, Erwin CW, Schuster JM, et al. Baeps in infants with myelomeningocele and later development of Chiari II malformation-related brainstem dysfunction. Dev Med Child Neurol 1994;36:707-715. 